DOI: https://doi.org/10.46296/ig.v111.0002

\title{
OPTIMIZACIÓN DEL PROCESO DE DESCARGA DE ACEITE CRUDO DE PALMA EN LAS EXTRACTORAS DE LA ZONA 4 DEL ECUADOR
}

\section{OPTIMIZATION OF THE PROCESS FOR DISCHARGING CRUDE PALM OIL IN THE EXTRACTORS IN ZONE 4 OF ECUADOR}

\author{
Andrade-Bastidas Paulo ${ }^{1 *}$ \\ ${ }^{1}$ Universidad Laica Eloy Alfaro de Manabí, ULEAM. Manta, Ecuador. \\ *Correo: pauloandradebastidas@gmail.com
}

\begin{abstract}
Resumen
En la actualidad, las empresas que procesan productos oleaginosos utilizan como materia prima principal la Palma cruda, de la cual se derivan la Palma RBD, oleína, estearina, ácidos grasos, entre otros. Razón por la cual las extractoras que cubren la Zona 4 del Ecuador tienen una gran demanda en la venta de este producto, cuya transportación se realiza mediante tanqueros. No obstante, la entrega de este producto genera inconvenientes, debido a que el producto debe mantenerse caliente durante su almacenamiento en los tanqueros para evitar las pérdidas por solidificación del producto. Esto último hace que la empresa extractora sufra una pérdida económica. Esta cantidad no afecta a los clientes, ya que cancelan el valor por la cantidad que reciben, pero sí afecta a las extractoras porque ellas ganan económicamente por la cantidad entregada. Basándonos en este análisis se realizó un diseño experimental para poder determinar y cuantificar las cantidades mensuales de producto no descargado y lo que representa en dinero. Se plantea en este trabajo una propuesta para que los tanqueros tengan un sistema interno de calentamiento (serpentín), y este pueda permitir que el producto esté listo para las descargas, logrando así un mayor aprovechamiento del producto entregado.
\end{abstract}

Palabras clave: extractoras, aceite crudo, tanqueros, descargas, calentamiento.

\begin{abstract}
Currently, companies that process oilseed products use raw palm as their main raw material, from which RBD palm, olein, stearin, fatty acids, among others, are derived. Which is why the extractors that cover Zone 4 of Ecuador have a great demand in the sale of this product, whose transportation is carried out by tankers. However, the delivery of this product generates inconveniences, because the product must be kept warm during storage in the tankers to avoid losses due to solidification of the product. The latter causes the extraction company to suffer an economic loss. This amount does not affect customers, since they cancel the value for the amount they receive, but it does affect the extractors because they earn financially for the amount delivered. Based on this analysis, an experimental design was carried out to determine and quantify the monthly amounts of product not downloaded and what it represents in money. A proposal is put forward in this work so that the tankers have an internal heating system (coil), and this can allow the product to be ready for unloading, thus achieving a greater use of the delivered product.
\end{abstract}

Keywords: extractors, crude oil, tankers, discharges, heating.

Información del manuscrito:

Fecha de recepción: 11 de septiembre de 2017

Fecha de aceptación: 20 de diciembre de 2017

Fecha de publicación: 10 de enero de 2018 


\section{Introducción}

La excelencia de una organización está basada en las ideas de mejora con las que puede aportar en cada uno de los procesos, de acuerdo a las necesidades y a las situaciones actuales en las que se encuentren, siendo las extractoras empresas transnacionales que permanentemente buscan realizar proyectos de mejora de sus procesos y de crecimiento empresarial, lo que las convierte en empresas de mayor proyección en el Ecuador.

Estas empresas realizan descargas de productos oleaginosos como Palma, Soya, Girasol, canola, entre otros que son procesados $y$ exportados.

Según Guevara-Cárdenas (2013) en la investigación sobre la evolución de la producción de aceite de palma y su incidencia en las exportaciones no tradicionales se analiza, en primera instancia, el rol que ha tenido la producción y exportación del aceite de palma en el Ecuador.

Los estudios realizados por Castillo González (2007) mencionan que la tecnología aplicada en la producción de aceites comestibles en el Ecuador es muy competitiva, por ello existe un gran número de plantas industriales dedicadas al procesamiento de oleaginosas como la palma africana.

Por lo tanto, se requiere de un sistema de control del proceso eficaz que permita minimizar la pérdida de aceite y producir productos de la más alta calidad. El papel del departamento de control del proceso es proveer información crítica a la administración para garantizar que se cumplan los objetivos del proceso. Este artículo pondrá en relieve algunos de los factores que afectan el rendimiento de aceite y la calidad del aceite de palma crudo y del Palmiste. También se discuten las medidas de control de calidad y del proceso requeridas para lograr los objetivos del proceso (Fedepalma, 2014).

El presente trabajo se enfoca en las descargas del aceite crudo de palma que se realizan diariamente. Este proceso se lo hace mediante tanqueros de 28 toneladas de capacidad aproximadamente; el problema que existe en las descargas es que los tanqueros no descargan por completo el producto y éste queda adherido a las paredes 
debido a que se solidifica rápidamente, y por ende esa cantidad no descargada representa un costo. El cuello de botella fue la falta de calentamiento al momento de descargar la palma, bajo este problema una vez identificado se pudo realizar el estudio y realizar la propuesta de mejora (AlvaradoTobías, 2010).

La importancia de esta mejora se centra en que busca desarrollar mecanismos que permitan mejorar el desempeño de los procesos, es decir, optimizarlos en función de la reducción de costos y el incremento de la productividad y calidad. El alcance de la investigación es que la descarga de aceite crudo de palma sea más eficaz, y que permita obtener un mejor rendimiento en la entrega al cliente a diario, mejorando la producción.

El tipo de investigación es concluyente causal, ya que se realizó un diseño experimental para determinar la principal opción que permita mejorar la descarga, después se evaluarán los costos del proyecto para determinar la viabilidad de la implementación de la propuesta económica.
Los tanqueros que realizan este tipo de descarga ganan por la cantidad descargada, y es por eso que se va proponer que los tanqueros tengan un sistema interno de vapor (serpentín) que permita tener el producto listo para ser entregado en su totalidad, y por ende generar mayores ganancias.

\section{Metodología}

Como se ha mencionado en el presente manuscrito, se evaluó cómo mejorar la eficiencia en la descarga de aceite que realizan las extractoras de la Zona 4 del Ecuador para poder obtener mayores beneficios.

La metodología utilizada para la investigación es experimental-de campo, puesto que el experimento se realizó en tiempo real plasmando observaciones y las respectivas anotaciones, además se manipularon variables con el fin de determinar la problemática del proyecto.

Ya que definimos una nueva modalidad de trabajo que genera conocimiento, que produce cambios, y mediante la evaluación e investigación se obtuvieron ideas de mejora que se reflejan en los 
resultados obtenidos al final de este proyecto.

La profundidad de la investigación se plantea al momento de usar sus variables dependientes e independientes, además el tipo de relación que mantienen. La amplitud macroeconómica de la investigación se basa sobre la agregación a nivel de la Zona 4 del Ecuador. En lo que respecta al tipo de fuente utilizada, se considera secundaria, debido a que se revisaron estudios previos sobre el tema, así como datos estadísticos. El carácter de la investigación es cuantitativo, por las cifras de producto y colocaciones al sector privado.

En resumen, la investigación propuesta es una contrastación empírica, con un alcance de nivel exploratorio y explicativo que permitirá ampliar los conocimientos relacionados a la problemática de las variables mencionadas.

\section{Resultados y discusión}

Según Uribe-Santos (2010) la agroindustria del aceite de palma en el mundo es una de las actividades económicas de mayor proyección.
La industria aceitera del Ecuador se basa exclusivamente en dos tipos de oleaginosas, como son la palma africana y la soya. Sin embargo, con el fin de ofertar otro tipo de aceites el país importa anualmente alrededor de 120.000 toneladas de aceite de oliva, aceite de canola, aceite de girasol, tanto para la industria aceitera nacional; así como, para el mercado gourmet (Pilco, 2015).

\subsection{Análisis, interpretación y discusión de resultados}

Tomando a la empresa La Fabril SA como uno de los clientes de las extractoras de la Zona 4 del Ecuador, presentamos a continuación cantidades no descargadas donde se evidencia una diferencia de peso, que significa las cantidades que fueron despachadas desde las extractoras y recibidas en las empresas.

A continuación, en la tabla 1 se muestran los resultados del registro de pesos de los tanqueros que descargan en La Fabril SA, en donde se pueden evidenciar el peso de entrada, peso de salida, peso neto, peso diferencia enviado y diferencias de peso, según el sistema de báscula empleado en esta empresa. 
Tabla 1.

Registro de pesos de los tanqueros que ingresan a planta en $\mathrm{Kg}$

\begin{tabular}{|c|c|c|c|c|c|}
\hline Extractoras & Peso de entrada & Peso de sallda & Peso neto & Peso diferencla envlado & Dlferencla de Peso \\
\hline Palmisa & 53,620 & 19,810 & 33,810 & 34,770 & -960 \\
\hline $\mathrm{Palm} / \mathrm{sa}$ & 53,590 & 19,850 & 33,740 & 34,130 & -390 \\
\hline Energy \& Palma & 48,130 & 17,400 & 30,730 & 31,020 & -290 \\
\hline $\mathrm{Palm} \mid \mathrm{sa}$ & 53,660 & 19,890 & 33,770 & 34,050 & .280 \\
\hline Energy \& Palma & 50,430 & 17,200 & 33,230 & 33,490 & -260 \\
\hline Compa fía industrial & 51,600 & 17,570 & 34,030 & 34,290 & -260 \\
\hline Compa fila industrial & 50,240 & 16,710 & 33,530 & 33,770 & -240 \\
\hline Palmisa & 53,180 & 19,610 & 33,570 & 33,810 & -240 \\
\hline Compahila industrial & 50,610 & 18,070 & 32,540 & 32,760 & .220 \\
\hline Compahila industr|al & 51,960 & 18,430 & 33,530 & 33,740 & -210 \\
\hline $\mathrm{Pa} / \mathrm{m} / \mathrm{sa}$ & 52,440 & 18,570 & 33,870 & 34,080 & -210 \\
\hline Extractora Agricola & $49,8 \pi 0$ & 20,150 & 20,720 & 29,910 & -190 \\
\hline Palm|sa & 53,360 & 19,670 & 33,690 & 33,870 & -180 \\
\hline Palm/sa & 52,910 & 18,680 & 34,230 & 34,400 & -170 \\
\hline \begin{tabular}{|l} 
Extractora Agricola \\
\end{tabular} & 49,550 & 15,310 & 34,240 & 34,390 & -150 \\
\hline Extractora Agricola & 50,550 & 17,700 & 32,850 & 33,000 & -150 \\
\hline
\end{tabular}

Fuente: Sistema de báscula de la empresa La Fabril S.A.

El porcentaje de diferencia muestra las cantidades que quedaron retenidas dentro del tanquero, las cuales no pudieron ser descargadas, con un promedio de $275 \mathrm{Kg}$. La Fabril S.A., mediante un sistema Baan pesa los tanqueros cuando ingresan a la balanza y comparan con las guías enviadas desde las extractoras donde determinan los pesos de entrada, salida, neto y finalmente el producto no descargado.

Cuantificando el período del año 2015 son 396.01 toneladas que no se descargaron debido a problemas de solidificación.
Tabla 2.

Cantidades de producto no descargado por mes y año

\begin{tabular}{|c|c|c|}
\hline Meses & $\begin{array}{c}\text { Cantidades en Kilogramos } \\
\text { de Palma no descargada }\end{array}$ & $\begin{array}{c}\text { Cantidades en Tonelada } \\
\text { de Palma no descargada }\end{array}$ \\
\hline Enero & 33000 & 33 \\
\hline Febre ro & 32000 & 32 \\
\hline Marzo & 31000 & 31 \\
\hline Abril & 30000 & 30 \\
\hline Mayo & 32000 & 32 \\
\hline Junio & 35000 & 35 \\
\hline Julio & 33000 & 33 \\
\hline Agosto & 36000 & 36 \\
\hline Septiembre & 34300 & 34.3 \\
\hline Octubre & 33500 & 33.5 \\
\hline Noviembre & 33680 & 33.68 \\
\hline Diciembre & 32530 & 32.53 \\
\hline Totales & $396,010.00$ & 396.01 \\
\hline
\end{tabular}

Fuente: Sistema de báscula de la empresa La Fabril S.A.

Los tanqueros que ingresan a diario con aceite crudo de palma traen 28 toneladas aproximadamente, estos tanqueros una vez que descargan se retiran de inmediato hacia las extractoras para poder realizar otro 
viaje al día siguiente. Debido a que el aceite crudo de palma se solidifica de manera rápida, existen ocasiones en las que queda producto pegado en las paredes del tanquero y no es posible descargar toda la materia prima. Este producto no descargado representa un costo, ya que la tonelada de Aceite Crudo de Palma cuesta $\$ 700$.

En algunos experimentos comparativos simples puede conseguirse un mejoramiento significativo de la precisión, haciendo comparaciones de observaciones pareadas del material experimental. (Gutiérrez-Pulido \& De La VaraSalazar, s. f.).

Para poder interpretar y discutir resultados se realiza un diseño experimental, el cual será una comparación pareada que se presenta a continuación:

\subsection{Experimento}

Las extractoras de la Zona 4 del Ecuador (Manabí-Santo domingo) venden a las empresas oleaginosas el aceite crudo de Palma, y hacen las entregas mediante tanqueros de 28 toneladas de capacidad.

Debido a que dicho producto se empieza a solidificar a partir de los $25-30^{\circ} \mathrm{C}$ aproximadamente, éste debe de salir caliente desde las extractoras para poder llegar a una temperatura óptima de descarga $\left(45^{\circ} \mathrm{C}\right)$. Ya que en muchas ocasiones los tanqueros cuando llegan a su destino deben permanecer 102 días en las afueras de la planta, el producto se enfría y es allí donde se complica que la descarga sea total y no se pueda descargar toda la materia prima, quedando parte de la materia prima adherida en las paredes del tanquero.

Debido a que las extractoras ganan dinero de acuerdo a la cantidad descargada, se ha diseñado un nuevo método para aumentar la eficiencia en el proceso de descarga, el cual consiste en instalar un sistema de calentamiento interno (serpentín) que se utilizará para realizar el respectivo despegue de producto. En otras palabras, que los tanqueros cuenten con un sistema interno de calentamiento.

A continuación, se seleccionaron aleatoriamente 10 tanqueros de la muestra para verificar cantidades de peso en ambos métodos, obteniéndose los siguientes resultados. 
Pregunta: ¿Existe diferencia mermas y maximiza la producción? significativa que justifique el uso del Utilizar a 0,05.

nuevo método en el cual se reducen

Tabla 3.

Registro de pesos en $\mathrm{Kg}$.

Método actual

\begin{tabular}{|c|c|c|}
\hline $\begin{array}{c}\text { Acelte crudo de Palma } \\
\text { descargadoen planta en Kg }\end{array}$ & $\begin{array}{c}\text { Ace lte crudo de Palma envlado } \\
\text { desde extractora en Kg. }\end{array}$ & $\begin{array}{c}\text { Cantldad no } \\
\text { de scargada en Kg. }\end{array}$ \\
\hline 33,510 & 33,880 & -370 \\
\hline 33,880 & 34,120 & -240 \\
\hline 31,320 & 31,510 & -190 \\
\hline 34,340 & 34,490 & -150 \\
\hline 31,320 & 31,460 & -140 \\
\hline 33,680 & 33,820 & -140 \\
\hline 33,860 & 34,000 & -140 \\
\hline 31,970 & 32,100 & -130 \\
\hline 33,170 & 33,300 & -130 \\
\hline 34,230 & 34,350 & -120 \\
\hline
\end{tabular}

Método nuevo (serpentín)

\begin{tabular}{|c|c|c|}
\hline $\begin{array}{c}\text { Acelte crudo de Palma } \\
\text { de scargado en planta en Kg. }\end{array}$ & $\begin{array}{c}\text { Ace lte crudo de Palma envlado } \\
\text { de sde extractora en Kg. }\end{array}$ & $\begin{array}{c}\text { Cantldad no } \\
\text { descargada en Kg. }\end{array}$ \\
\hline 33,390 & 33,380 & 10 \\
\hline 32,810 & 32,780 & 30 \\
\hline 32,310 & 32,260 & 50 \\
\hline 30,590 & 30,570 & 20 \\
\hline 34,440 & 34,430 & 10 \\
\hline 34,020 & 33,990 & 30 \\
\hline 32,850 & 32,810 & 40 \\
\hline 33,105 & 33,080 & 25 \\
\hline 34,520 & 34,520 & 0 \\
\hline 33,800 & 33,770 & 30 \\
\hline & & \\
\hline
\end{tabular}

Fuente: Sistema de báscula de la empresa La Fabril S.A.

\subsection{Flujo de Efectivo Proyectado}

En el flujo de Efectivo Proyectado tenemos inicialmente en al año cero solo la inversión, que representa un valor de $\$ 109,962.90$. A partir del primer año tenemos un costo de oportunidad de $\$ 300,300.00$ menos los costos por mantenimiento
(\$3000), y restando el valor de la inversión inicial nos representa un flujo neto acumulado de $\$ 187,337.10$. Para los siguientes años se considera un aumento en la inflación del 5\% para los costos de mantenimiento. 
Tabla 4.

Flujo de efectivo proyectado en 5 años

\begin{tabular}{|c|c|c|c|c|c|c|}
\hline \multicolumn{7}{|c|}{$\begin{array}{l}\text { Flujo de Efectivo Proyectado } \\
\text { Empresa Extractora Río Manso }\end{array}$} \\
\hline Entradas: & Año 0 & Año 1 & Año 2 & Año 3 & Año 4 & Año 5 \\
\hline $\begin{array}{l}\text { Costo Oportunidad de } \\
\text { vender más (Ton./año) }\end{array}$ & & $\$ 300,300.00$ & $\$ 278,112.42$ & $\$ 257,564,16$ & $\$ 238,534.11$ & $\$ 220,910.09$ \\
\hline Total Entradas: & $\$$ & $\$ 300,300.00$ & $\$ 278,112.42$ & $\$ 257,564.16$ & $\$ 238,534.11$ & $\$ 220,910.09$ \\
\hline Desembolsos: & & & & & & \\
\hline Inversión Inicial: & $\$ 109,962.90$ & & & & & \\
\hline Costos por mantenimientos: & & $\$ 3,000.00$ & $\$ 3,150.00$ & $\$ 3,307.50$ & $\$ 3,472.88$ & $3,646.52$ \\
\hline Total Desembolsos: & $\$ 109,962.90$ & $\$ 3,000.00$ & $\$ 3,150.00$ & $\$ 3,307.50$ & $\$ 3,472.88$ & $\$ \quad 3,646.52$ \\
\hline Flujo Neto: & $\$(109,962.90)$ & $\$ 297,300.00$ & $\$ 274,962.42$ & $\$ 254,256.66$ & $\$ 235,061.24$ & $\$ 217,263.57$ \\
\hline Flujo Acumulado: & $\$(109,962.90)$ & $\$ 187,337.10$ & $\$ 462,299.52$ & $\$ 716,556.19$ & $\$ 951,617.42$ & $\$ 1,168,880.99$ \\
\hline
\end{tabular}

Fuente: Investigación propia.

La disminución de las entradas de efectivo se debe a la estimación promedio de la variación de los últimos 5 años, que estadísticamente es del $7,39 \%$ anual. Lo que quiere decir que, por cada año, el valor por tonelada de aceite crudo de Palma disminuye en un 7,39\%. Cabe señalar que este valor constituye la entrada de cada año consecutivo.

En el año cero no se considera ningún gasto de mantenimiento debido a que apenas se ha implementado el sistema, tampoco se observa ningún ingreso porque se está incursionando la inversión, por lo tanto, no es posible observar entrada de efectivo alguna.

Al término del primer año se empieza a recibir $\$ 300,300.00$, en este primer año se obtienen beneficios, descontando la inversión inicial y el costo de mantenimiento, puesto que de todos modos el flujo neto es positivo y se empieza a cuantificar un flujo acumulado.

Para los años siguientes: dos, tres, cuatro, y cinco se logra obtener un flujo acumulado de $\$ 1,168,880.99$.

La entrada de efectivo corresponde al costo de oportunidad, que es el 
beneficio de la alternativa que no se tomó, por lo tanto, se considera que la empresa extractora debería de ganar como ingreso bruto $\$ 187,337.10$ al término del año uno, $\$ 462,299.52$ al término del año dos, y así finalmente $\$ 1,168,880.99$ para el año cinco.

Nótese que se está considerando una pérdida del $7,39 \%$ porque el precio de la tonelada de Palma cruda va descendiendo en promedio durante los últimos 5 años, y esto hace que los flujos de entrada futuros tengan una disminución mínima.

\subsection{TIR}

La tasa interna de retorno (TIR) es utilizada en el rendimiento de presupuesto del capital para poder comparar la rentabilidad de las inversiones (García et al., 2010).

De acuerdo al Flujo de Efectivo calculado, el TIR fue del $262,55 \%$, lo que indica que el proyecto es total y absolutamente viable.

Afanador et al., (2012) mencionan que la TIR se puede utilizar como indicador de la rentabilidad de un proyecto, ya que a mayor TIR se espera una mayor rentabilidad; de esta forma se utiliza como uno de los criterios para decidir sobre la aceptación o rechazo de un proyecto de inversión. La TIR se compara con una tasa mínima o tasa de corte, el coste de oportunidad de la inversión (si la inversión no tiene riesgo, el coste de oportunidad utilizado para comparar la TIR será la tasa de rentabilidad libre de riesgo). Si la tasa de rendimiento del proyecto, expresada por la TIR, supera la tasa de corte, se acepta la inversión; caso contrario, se rechaza. En este caso, se obtuvo una TIR equivalente a $263 \%$.

\subsection{TMAR}

Para demostrar que el proyecto es viable se calculó la TMAR (Tasa Mínima Atractiva de rendimiento), es decir, cuanto es lo mínimo que el accionista desea ganar por implementar este proyecto.

TMAR: Tasa mínima atractiva de retorno que espera el accionista o inversionista de un proyecto, que, en este caso, en conversaciones con el accionista bordea el $50 \%$.

\subsection{VAN}

Si el VAN es mayor a cero, el proyecto es conveniente o aceptable y significa que el proyecto gana más que el interés de oportunidad. Si el 
VAN es igual a cero, el proyecto es indiferente y significa que la rentabilidad del proyecto es la misma que la del interés de oportunidad. Si el VAN es menor a cero, el proyecto no es conveniente o aceptable y significa que el proyecto gana menos que el interés de oportunidad.

El cálculo para el VAN se realizó al $50 \%$, fundamentado bajo el criterio que el accionista espera de rendimiento, obteniéndose así un VAN de $\$ 360,820.62$

\section{Conclusiones}

Se comprobó experimentalmente que con el sistema interno de calentamiento el proceso de descarga se realiza de forma más eficiente, lo cual representará mayores ingresos en ventas para las extractoras.

En el flujo de efectivo proyectado se obtiene para el año 5 un flujo neto acumulado de $\$ 1,168,880.99$; lo que sugiere que el proyecto sea considerablemente viable.

La TMAR que el inversionista espera es del $50 \%$, lo cual supera las expectativas, ya que la TIR calculada fue del $263 \%$.
Finalmente, la relación beneficiocosto del proyecto indica que, por cada dólar que la empresa invierta para la instalación del sistema interno de calentamiento en carrotanques, obtendrá un beneficio de $\$ 85.88$, lo cual permite concluir que el proyecto es viable.

\section{Bibliografía}

Afanador, J., González, D., Gómez, R., \& Tovar, D. (2012). Cálculos Tas de Retorno. Obtenido de: http://www.monografias.com/t rabajos101/calculos-tasaretorno-proyectounico/calculos-tasa-retornoproyecto-unico.shtml.

Alvarado-Tobías, M. (2010). Evaluación del proceso de clarificación en la planta de beneficio Palmagro S.A. Colombia: Universidad Nacional de Colombia.

Castillo-González, A. (2012). Tecnología aplicada en la producción de aceites comestibles en el Ecuador

Fedepalma. (2014). La planta extractora de aceite de palma: control del proceso. Revista Palmas, 19.

García-Mujica, R., BenavidesCedeño, G., \& AlcívarCedeño, U. (2018). Distribución y análisis financiero de una planta de producción de ácido láctico. 


\section{Revista científica \\ multidisciplinaria arbitrada \\ YACHASUN, 2(3), 20-28. \\ https://doi.org/10.46296/yc.v2 \\ i3.0011}

Guevara-Cárdenas, I. (2013). Evolución de la producción de aceite de Palma.

Gutiérrez-Pulido, H., \& De La VaraSalazar, R. (s.f.). Análisis y diseño de experimentos. Guadalajara-México, GUA, México: Mc Graw Hill.
Pilco-Saca, G. (2015). Optimización del proceso de extracción de aceite de Tungurahua (Oenocarpus bataua) en función del rendimiento. Ambato-Ecuador: http://repositorio.uta.edu.ec/js pui/handle/123456789/9366.

Uribe-Santos, G. (2010). Extracción de Palma y Palmiste. Colombia: Fedepalma. 